\title{
Avaliação Do Risco De Sangramento Maior Com Anticoagulantes Orais Antagonistas Da Vitamina K Em Pacientes Com Fibrilação Atrial.
}

\author{
Da Silva, A.L.F.; Pivatto Júnior, F.; Bezerra, I.V.; Pires, L.M.; Blaya, M.B.; \\ Scheffel, R.S.; \\ Apresentador: André Luís Ferreira da Silva
}

\section{Resumo}

Introdução: a fibrilação atrial é um dos principais fatores de risco para acidente vascular cerebral (AVC). O uso de escores de risco para sangramento maior e AVC nos pacientes portadores dessa arritmia auxilia na avaliação do risco versus benefício da terapia de anticoagulação oral, que reduz significativamente o risco dessa complicação. Objetivos: descrever o percentual de pacientes anticoagulados por fibrilação atrial não-valvular com alto risco de sangramento maior em um ambulatório específico de controle de anticoagulação através do escore HASBLED, assim como identificar possíveis fatores de risco de sangramento modificáveis e comparar o risco de sangramento maior versus risco de AVC. Metodologia: estudo de coorte retrospectivo incluindo pacientes do Ambulatório de Anticoagulação do Serviço de Medicina Interna do HCPA. A análise descritiva foi realizada a partir da distribuição de frequência absoluta e relativa, para as variáveis qualitativas, e através da média/mediana e desvio-padrão/intervalo interquartil, para as quantitativas. Resultados: foram estudados 63 pacientes, com idade média $( \pm d p)$ de $74,3 \pm 10,9$ anos. A mediana (25-75\%) do escore HAS-BLED foi de 2(1-3) pontos, sendo ? 3 (alto risco) em 19(30,2\%) pacientes. Os fatores de risco modificáveis mais prevalentes foram TP
INR lábil (36,5\%) e o uso de drogas concomitantes $(30,2 \%)$. O risco de sangramento maior baseado no escore HAS-BLED foi superior ao de AVC em $3(4,8 \%)$ e $4(6,3 \%)$ pacientes, na comparação com o escore CHADS2 e CHA2DS2-VASc, respectivamente. Conclusões: o percentual de pacientes com alto risco de sangramento maior foi de $30,2 \%$, sendo identificados TP INR lábil e uso de drogas concomitantes como os fatores de risco modificáveis mais prevalentes. Além disso, verificou-se que o risco de sangramento maior foi superior ao risco de AVC em apenas $6,3 \%$ dos casos. $\mathrm{O}$ uso de escores de risco auxilia no embasamento da decisão clínica de início/ manutenção da anticoagulação nesses pacientes.

\section{Referência:}

Da Silva, A.L.F.; Pivatto Júnior, F.; Bezerra, I.V.; Pires, L.M.; Blaya, M.B.; Scheffel, R.S.; Avaliação Do Risco De Sangramento Maior Com Anticoagulantes Orais Antagonistas Da Vitamina K Em Pacientes Com Fibrilação Atrial.. In: II Congresso

Brasileiro de Medicina Hospitalar - II CBMH [= Blucher Medical Proceedings, vol.1, num.5] São Paulo: Editora Blucher, 2014. p.79

DOI 10.5151/medpro-II-cbmh-077 